\section{REPORT ON ANARCOTINE.}

\section{By NUSSERWANJI SURVEYOR, M.D., M.A.Bombay,} M.R.C.P.LoND.

THE work was undertaken to determine the influence of the alkaloids of opium, chiefly anarcotine, on metabolic changes produced in animals. At one time, before the introduction of quinine, this alkaloid was largely used as an antiperiodic. In India, however, as stated by Sir William Roberts in his presidential address before the Pharmacological Section of the British Medical Association, ${ }^{1}$ it has now been altogether discarded.

The general impression about the value of the drug at present is that it is a harmless principle of opium. Experiments were performed to determine the effect of this drug in diminishing or increasing metabolic changes in dogs. Blood-pressure experiments were also performed on dogs; however, it was found that a subcutaneous dose of the chloride of anarcotine, even to the extent of $4 \mathrm{~g}$., had no effect on the blood pressure or the heart. The respiratory movements of the animals were not altered. Respiratory changes of gases before, during, and after injection of the chloride were studied in mice. It was found that a subcutaneous injection of $0.016 \mathrm{~g}$. of anarcotine chloride per body-weight of $100 \mathrm{~g}$. produced diminution of the amount of $\mathrm{CO}_{2}$ given off by the animal; the amount of oxygen absorbed was also slightly diminished. The respiratory ratio was not much altered. The animals lost weight during administration of the drug, which was soon regained on stopping it.

Administration of pure anarcotine to dogs in doses of I g. produced vomiting within half an hour. The smallest dose required to produce this effect was $0.5 \mathrm{~g}$. per body weight 5 kilogrammes.

Anarcotine chloride ( 1 g.) produced slight retching in one dog, while subcutaneous injection of the salt up to $4 \mathrm{~g}$. in an anæsthetised animal did not produce any effect

It is curious that a perfectly tasteless alkaloid like anarcotine should produce vomiting, while its chloride, which is extremely bitter in taste, can be tolerated to a greater extent.

The chloride was prepared from the alkaloid by the action of just enough hydrochloric acid. ' The resulting salt was dissolved in distilled water, filtered, evaporated down to dryness, dissolved in chloroform, and crystallised out from the chloroform solution by the addition of anhydrous ether. The crystals were redissolved, and recrystallised salt used for the experiments.

As regards the effect of the alkaloid on metabolism, great difficulty was met with in the matter of diet. Some dogs refused to take the ordinary dried meat, so it was ultimately decided to feed them on sterilised meat. Minced meat was weighed in small beakers, and these sterilised at $120^{\circ} \mathrm{C}$. in the autoclave. During administration of the drug the animals lost weight, and the nitrogen absorbed was found to be diminished; in one case it fell to one-third the normal amount. The amount of fat absorbed was also diminished to some extent.

The amount of water excreted as urine did not appear to be regularly influenced, however; as a rule it was increased, and on some occasions it was found that the total amount of water excreted both as urine and in fæces was in excess of the amount taken on the corresponding day. This observation requires further study before one can venture to state anything definite about it. There was slight diminution in the amount of nitrogen excreted by the kidneys.

The metabolic changes after the administration of hydrochlorate of morphine were studied in one dog. The dose given was $0.05 \mathrm{~g}$. to $0.02 \mathrm{~g}$. per 5 kilo. body weight. In this case the animal was very restless; it took very little food, and lost weight; the amount of weight lost, however, did not correspond to the nitrogen and fat excreted during the same period. In fact, although very little food was taken, the weight lost was not considerable. This can be accounted for in this case, and also in cases of anarcotine experiments, by the fact that the amount of nitrogen excreted by the kidneys was also diminished to a great extent.
Further work on the subject is being carried on. Most of the above work was done at the Pathological Laboratory of University College, London, in collaboration with Dr. Vaughan Harley, to whom my best thanks are due, and also to Professor Victor Horsley, in whose laboratory the work was done. The anarcotine used for these experiments was kindly supplied by Dr. A. R. W. Sedgefield, Factory Superintendent, Opium Factory, Ghazipur, North-West Provinces (India), to whom best thanks are due.

\section{CLINICAL REMARKS ON A CASE OF COMPLETE CLOSURE OF THE BOWELS FOR TWO MONTHS FROM IMPACTION : NO URGENT SYMPTOMS : TREATMENT : RECOVERY.}

BY THOMAS OLIVER, M.D., F.R.C.P.,

Physician to the Royal Infirmary, Newcastle-upon-Tyne.

OBstinate constipation is of such common occurrence in females that it is difficult to say how long their bowels may be closed without any serious symptoms following. I know of one case where no stool was passed for six weeks, but in the case about to be reported the bowels were not moved for eight weeks. As to the accuracy of the length of time of the closure, there is little doubt. The district nurse who attended the patient bears testimony to six weeks having passed without a stool, and both in her own belief and that of the patient there had been no movement for a fortnight previously. In cases of intestinal obstruction the urgency of symptoms depends on whether the blockage is complete or incomplete, whether it has been slowly or rapidly developed, and whether it is accompanied or not by inflammation. It is well to remember that a patient may complain of diarrhœa or the passage of thin liquid discharges from the bowel-nay, may even give a history of having taken a pill daily for yearsand yet neither of these is any proof whatever that the lower part of the bowel is being thoroughly unloaded. In spite of all this, the rectum may be found impacted with fæces.

The patient who is the subject of my remarks was a married woman aged 53 years, without a family. She was admitted into the infirmary on May 27th, 1896, complaining of not having had her bowels moved for two months. She had been married twenty years, and had ceased menstruating at the age of 48 . There was nothing in her family or social history that calls for comment. For the last two years she had suffered from indigestion, and had experienced a sense of swelling and flatulence after eating. Vomiting has been absent all through her illness. Until a little more than a year ago there was no constipation, the bowels being then moved daily and apparently quite satisfactorily. During the first six months of 1895 , however, she had to to take aperient medicine pretty frequently. Since then the bowels have acted very irregularly, sometimes not for a week at a time. There has been no pain, but occasionally headache. Gradually the constipation became worse, and for the last eight weeks there has been no movement of the bowels at all. Occasionally she has passed flatus, and latterly she has been conscious of the simultaneous escape of a very small quantity of a thin dirty brown fæcal liquid from the rectum. For the last six months she has had uneasy sensations of a " bearingdown" character, and of pain about the anus and rectum.

At the time of her admission she was emaciated, sharpfeatured, dull mentally, melancholic, and extremely obstinate. Her marked emaciation suggested the probable existence of malignant disease, but neither at that date nor two months afterwards were there any physical signs of cancer. Un the contrary the subsequent history of the case pointed to its non-existence.

On admission : Chest, heart, and lungs are healthy. Abdomen : Theabdomen is uniformly flat and even, except in theiliac fossæ, where there is some bulging. An occasional peristaltic movement of the intestine is observed. On palpation there can be seen and felt in the left iliac region passing towards the pelvis a hard but doughy mass. Above this and extending up the line of the descending colon there can be seen and felt numerous masses about the size of a walnut, which can 
be moved freely, and are easily altered in shape by pressure of the finger. In the neighbourhood of the cæcum similar masses are observed. Rectal examination is made with difficulty, partly on account of pain, but largely from unwillingness of the patient to submit to it. The rectum is full of extremely hard fæces. So fully loaded is the rectum that it is almost impossible to pass a finger even into the vagina, and quite impossible to get beyond the bulging to examine the os uteri. Subsequently, however, and on several occasions this was done, as also the rectum carefully explored for malignant disease, but without any being detected.

As the patient obstinately refused to have enemata administered, or any local treatment carried out on account of pain, she was put under the influence of an anæsthetic (ether), and thus my house-phyiscian (Dr. Percival Davidson) succeeded in breaking up and removing, by scooping and washing out, several masses of hardened fæces. Ten pints of soapy water in divided quantities. were injected into the bowel, and by this means, as well as by kneading the abdomen, many hard scybalous masses were discharged. She slept well that evening, and next day the bowels were moved of their own accord. There could still be felt through the abdominal wall several discrete masses in the intestine, but by means of mist. sennæ co. internally and a large enema of soap and water, with some glycerine and turpentine, a large stool was brought away. The urine at this stage was deep ambercoloured and acid; it had a specific gravity of 1,027 , and did not contain either albumen, sugar, or indican.

In a few days after we had got the bowels thoroughly emptied, and were able to keep them moved by a small aperient and tonic pill administered twice a day, her mental stupor disappeared, and her temper and disposition improved. It was remarkable how the toxæmic symptoms subsided as absorption from intestinal contents became less possible. From this date onwards everything became more natural with the patient, so that instead of persisting in her positive refusal to take food, she now began to eat and with avidity. She made, thanks to Dr. Davidson's attention, a good recovery. Before leaving the infirmary she had gained upwards of a stone in weight. Such was the stimulus to nutrition that she gained six pounds in weight in one week. This fact, along with the improvement in her general health, mental as well as physical, and the complete absence of any pathological lesion, obliges us to regard the case as one of intestinal obstruction from impaction, lasting fully eight weeks, a period which at first sight seems almost incredible.

\section{INTUSSUSCEPTION : RECOVERY BY GANGRENE: NECROPSY THREE YEARS LATER.}

By WM. RUSHTON PARKER, M.A., M.D.CANTab., Honorary Surgeon to the Kendal Hospital.

J. S., a plumber, then aged 27, began to suffer in the latter part of April, 1893, from colic and obstinate constipation. The pain gradually increased, becoming at times severe; the constipation occasionally alternated with slight diarrhca; the motions were often mucous and bloody, and attended with considerable tenesmus; the tongue became dry; there was considerable thirst and no appetite; there was troublesome flatulence with very little expulsion of tlatus, no great tympanites, no vomiting, and nothing to be made out by palpation or rectal examination.

The patient was kept on peptonised milk given in frequent small quantities, and sedatives were frequently administered. After a month's illness he passed per anum a nearly complete cylinder of intestine, to or 12 inches in length. For two months subsequently he was still laid up with general weakness, flatulent dyspepsia, great discomfort of the bowels, constipation alternating with fœtid loose motions, and frequently some pain. By the first week in August he was out-of-doors, and soon completely recovered and returned to work, remaining perfectly well for the subsequent three years.

In the latter part of April, 1896, he again began to suffer from colic and obstinate constipation, followed this time by persistent vomiting; tympanites gradually increased, but never became very marked; borborygmi increased; peristalsis became easily visible; he often passed flatus; ene- mata occasionally brought away bits of fæces, looking as if squeezed through a slit; nothing could be learnt from palpation; the tongue became dry and brown. For a couple of weeks he was fed on small quantities of milk and beef-tea, and af terwards restricted to water and nutrient suppositories. Hypodermic injections of morphine with atropine were frequently administered. During the latter half of May the bowel became paralysed, visible peristalsis ceased, borborygmi diminished, constipation became absolute, so that not even flatus was passed, and vomiting ceased. The pain remained severe and almost constant, though kept in abeyance by morphine. There was great thirst; the tongue remained dry and brown; the pulse quickened up to 144, becoming simultaneously weaker; the urine was in moderate quantity and deposited urates; tympenites remained moderate; an occasional enema still brought away small bits of squeezed fæces, like dried currants; great emaciation and weakness set in, and the patient died

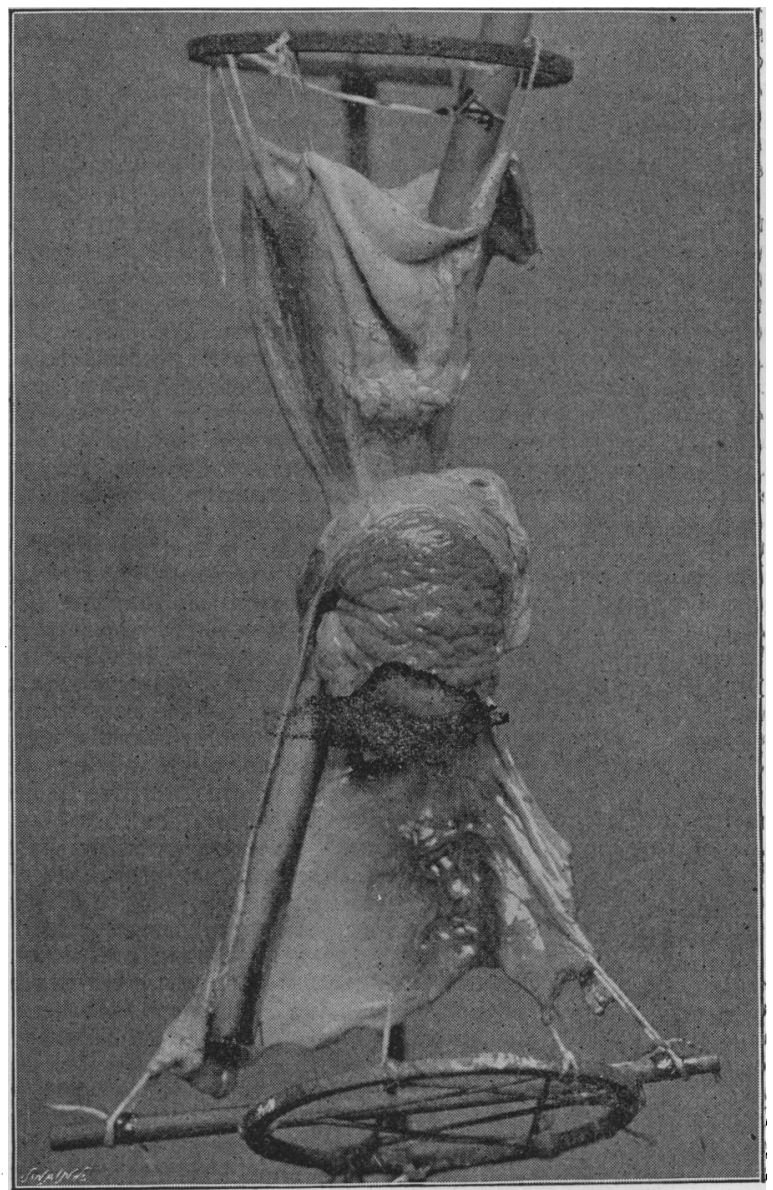

Invagination of descending colon. Intussusceptum protrudes 3 inches: within its sheath, and terminates in an ulcer, from which over ro inches of gut had sloughed three years previously; cane passed in
natural channel of gut by side of excentric intussusceptum.

At the necropsy, made almost immediately after death, general peritonitis was found, with tlakes of lymph in the furrows between adjacent coils of intestine. The stomach and bowels were moderately distended. In the lower part of the descending colon an invagination was found, springing from one side of the bowel, so that the solid tough intussusceptum lay, not concentrically with its sheath, but excentrically, projecting 3 inches within its sheath (like a hypertrophied uterine cervix projecting into the vagina), and terminating at its free extremity in a foul ulcer, from which a piece of bowel had evidently sloughed away. By the side of the intussusceptum it was not difficult to pass a finger from the dilated and hypertrophied bowel above to the narrower and 